\title{
Clinical Applications of Late Gadolinium in Children
}

\author{
Moises Rodriguez-Gonzalez., $\mathrm{MD}^{1^{*}}$, Ana Castellano-Martinez ${ }^{2}$ \\ ${ }^{I}$ Pediatric Cardiology Department of Hospital Universitario Puerta del Mar, Cadiz, Spain \\ ${ }^{2}$ Pediatric Department of Hospital Universitario Puerta del Mar, Cadiz, Spain
}

*Corresponding Author: Moises Rodriguez-Gonzalez, Department of Pediatrics, Hospital Universitario Puerta del Mar, Ana de Viya Avenue 34, Cadiz, Spain, Email: doctormoisesrodriguez@ gmail.com

\begin{abstract}
Cardiac magnetic resonance (CMR) imaging has recently emerged as one of the most promising available imagines techniques with an increasing role in the diagnostic process in pediatric cardiology. Noninvasive tissue characterization avoiding ionizing radiation is the main advantage respect to other cardiac imaging techniques. CMR with late gadolinium enhancement $(L G E)$ sequence is useful in characterizing several heart diseases based on the pattern and distribution of enhancement, and offers information regarding diagnosis and prognosis. In the pediatric setting the main applications of LGE include a wide and heterogeneous range of cardiac disease, including myocarditis, pericarditis, Kawasaki disease, cardiomyopathies, congenital heart disease in pre and post surgery stage and cardiac tumours. In this article we review different clinical applications of LGE sequence in children, highlighting its potential in the noninvasive management of a great variety of heart disease in this population.
\end{abstract}

Keywords: Late gadolinium enhancement; Cardiac magnetic resonance; Heart disease; Children.

\section{Cardiac Magnetic Resonance (CMR)}

Echocardiography is the initial imaging technique to assess cardiac function and structure in pediatric cardiology but its ability to provide specific tissue characterization is modest. Unlike echocardiography, CMR has the ability to obtain high-resolution tomographic imaging of the heart providing high-quality images that allow a comprehensive cardiological assessment in children, adding important data in terms of anatomy, hemodynamics and function[1]. In addition CMR can characterize myocardial tissue, and assess myocardial viability and perfusion[1]. Also it is a noninvasive imaging modality and it avoids ionizing radiation of cardiac catheterization and CT scan [2]. Thus CMR imaging has recently emerged as one of the most promising available imagines techniques with an increasing role in the diagnostic process in pediatric cardiology.

\section{Tissue Characterization}

The main advantage of CMR in comparison to other imaging techniques is the possibility to perform non-invasive tissue characterization. CMR usually differentiates well 3 cardiac components: fat, edema and scar/fibrosis. Proton relaxation times (T1, T2, and $\mathrm{T} 2 *$ ) reflect the composition of water protons in tissues. T1- and
T2-weighted images are specific sequences designed to visually discriminate tissues according to their T1 or T2. Analysing the same zone on $\mathrm{T} 1$ and $\mathrm{T} 2$ maps can recognize each tissue (fat low $\mathrm{T} 1 /$ high $\mathrm{T} 2$; fibrosis high $\mathrm{T} 1$; edema high T1/ high T2). The use of a contrast agents such as gadolinium is recommended to increase the contrast between normal and pathologic myocardium $[1,3]$.

\section{LATE GAdOLINIUM ENHANCEMENT (LGE; FIGURE 1)}

Gadolinium is an extracellular agent diffusing from intravascular to extravascular space without passing through the myocytes cell membranes, which accumulates in regions of interstitial expansion [4-6]. Normal myocardium has densely packed myocytes $(85 \%$ of myocardial volume) and very little extracellular space. In some pathological conditions the effective extracellular space increases, which leads to retention of gadolinium within the myocardium. Gadolinium-based contrast agents usually pass in and out of normal myocardium rapidly, but in abnormal tissues with increased extracellular space, such as scar/fibrosis, they pass slowly leading to prolonged contrast retention that cause LGE. 

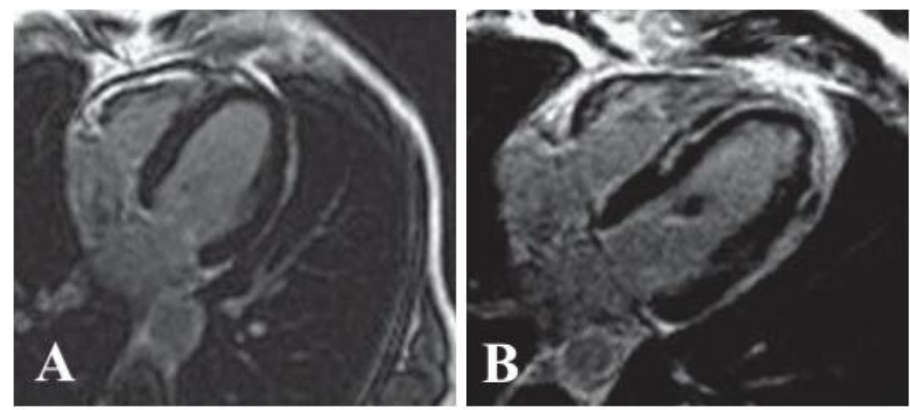

Figure1. LGE. A. CMR prior to the administration of gadolinium. B. CMR T1-weighted sequence after 20 min of administration of gadolinium with septal midwall and apical subepicardial enhancement.

LGE sequences are possible because of the property of gadolinium to shorten the T1relaxation and increase the signal intensity on T1-weighted images (hyperenhancement). The most widely used sequence for the evaluation of LGE is a two-dimensional T1-weighted, ECG gated, inversion recovery, spoiled gradient echo sequence[1].

LGE imaging is performed after an intravenous gadolinium injection $(0.1-0.2 \mathrm{mmol} / \mathrm{kg})$, when three post-contrast phases can be assessed: 1) First pass, is immediate and allows to visualize perfusion defects. 2) Early gadolinium enhancement, acquired at 90-120 s, detects thrombi, edema and microvascular obstruction post myocardial infarction. 3) LGE, acquired at 5 min-20 min, detects delayed contrast washout in areas of infarction, fibrosis or inflammation. Gadolinium-based contrast agents are nephrotoxic (nephrogenic systemic fibrosis), so they should be avoided in acute or chronic disease with eGFR $<30 \mathrm{ml} / \mathrm{min} / 1.73 \mathrm{~m} 2$. Caution should also be exercised in preterm neonates and infants because of renal
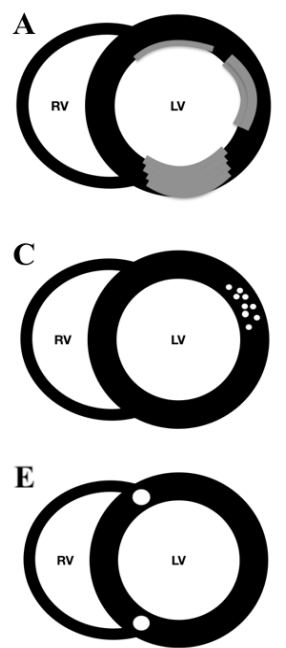

immaturity and potential eGFR $<30 \mathrm{ml} / \mathrm{min} / 1.73$ $\mathrm{m} 2$. Usually $0.2 \mathrm{mmol} / \mathrm{kg}$ when estimated glomerular filtration rate (eGFR) is $>60$ $\mathrm{ml} / \mathrm{min} / 1.73 \mathrm{~m} 2$ and $0.1 \mathrm{mmol} / \mathrm{kg}$ when eGFR is $30-60 \mathrm{ml} / \mathrm{min} / 1.73 \mathrm{~m} 2$.

\section{LGE PATTERNS (Figure 2)}

LGE patterns have been divided into ischaemic and non-ischaemic patterns $[4,7,8]$.

Ischaemic pattern: LGE always involve the subendocardium following a coronary territory. As the coronary occlusion time increases the LGE transmural extension to subepicardium increases.

Non-ischaemic pattern: LGE can present with different patterns that either not affect the subendocardium or not correspond to any coronary territory. The high-signal areas may affect the mid-wall or the epicardium, with either a diffuse or focal distribution. Nonischemic LGE patterns include mid-myocardial, subepicardial, fine interstitial, or diffuse subendocardial.

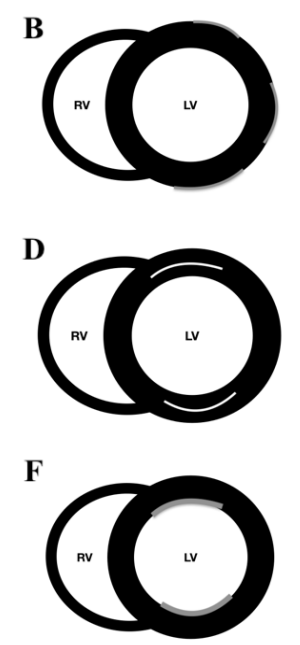

Figure2. Different LGE patterns. A. Ischemic pattern affect always subendocardial areas with varying degrees of transmural or subepicardial extension depending of the severity and the duration of the ischemic insult. B-F. Non-ischemic patterns, that affect usually non-subendocardial areas or if affect them, they do not follow a coronary artery distribution. (B, subepicardial pattern; C fine interstitial pattern; D midwall pattern; E HCM pattern; F diffuse subendocardial pattern). 


\section{Clinical Applications in Pediatrics}

LGE is one of the most widely used and investigated CMR technique for determining etiology as well as the prognosis in the setting of heart diseases. Enhanced (white) regions indicate areas of gadolinium accumulation and suggest the presence of inflammation, fibrosis, or scar tissue when specific T1- weighted pulse sequences are performed. The most important feature of LGE sequence is that it can differentiate well between ischaemic and nonischaemic patterns in different forms of both acquired and congenital heart diseases affecting children[3,9,10]. Although ischemic heart disease is rare in the pediatric setting when it occur a rapid differential diagnosis (usually viral myocarditis vs infarct in the context of chest pain with troponin elevation) and treatment are necessary highlighting the value of this technique. Also in the setting of both ischemic and not ischemic heart disease, the presence of myocardial fibrosis has important prognostic implications[4].

\section{MYOCARDIAL INFARCTION (FIGURE 3)}

Myocardial infarction (MI) is very rare in children. It is caused mainly by Kawasaki disease, anomalous coronary arteries or in the

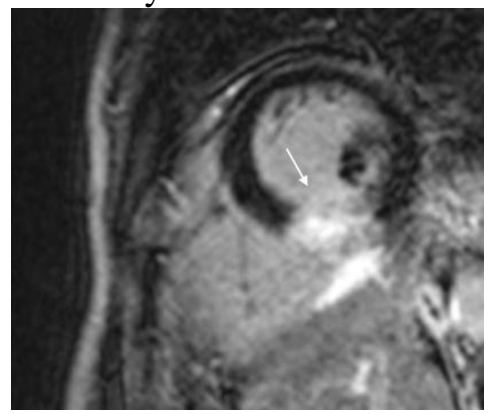

context of surgical correction of congenital heart disease, but also by congenital hypercholesterolemia, hyper-coagulation states, coronary dissection, vasculitis and embolus[3,11]. LGE is a very accurate technique for the detection of acute MI (LGE ischemic pattern) and the extent of the infarct peripheral zone, an area with both viable and non-viable myocytes. A larger peripheral zone has been related to an adverse prognosis with higher mortality at follow-up due to arrhythmia. LGE can also detect the ischemic area at risk after a coronary occlusion. This area will not show enhancement on LGE, helping to differentiate it from the infarcted zone. LGE can also offer some important prognostic data. The extent of enhanced myocardium correlates with biomarkers of myocardial necrosis and left ventricle ejection fraction at late follow-up in patients with acute MI. In chronic ischemic heart disease, the major contribution of LGE is to predict recovery of function after revascularization[4,9]. The presence and extent of LGE are strongly associated with the probability of improvement in contractility, with a low likelihood of improvement after revascularization as the transmural extent is increased.

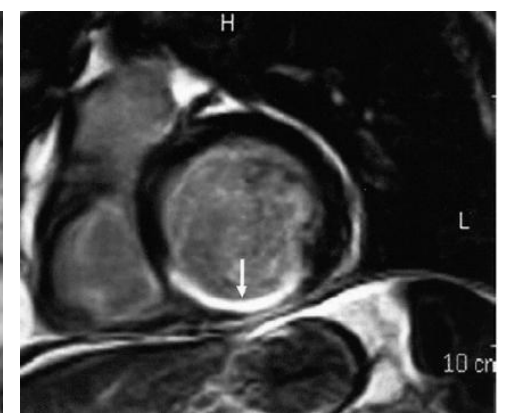

Figure3. LGE sequence showing ischemic pattern with complete (up) and partial (down) transmural extension of the inferior myocardial wall.

\section{MYOCARDITIS (FIGURE 4)}

Myocarditis is an inflammatory myocardial disease usually caused by an acute viral infection in children; it is one of the main causes of dilated cardiomyopathy and cardiac transplant in children. Biopsy is the diagnostic gold standard method; however because it is invasive and does not always give the diagnosis (the myocardial affectation is usually partial and patchy) it is reserved only for children with a severe course of the disease. In the clinical setting of acute myocarditis, CMR represents the best non-invasive diagnostic method; it allows for the assessment of inflammatory acute changes and degree of irreversible injury $[9,12,13]$. It also can avoid an initial cardiac catetherism in cases of previously healthy children with chest pain and elevated troponins, because myocardial infarct is so rare in paediatrics. Also it correlates well with histopathology. LGE sequences provide a distinctive pattern of subepicardial and mid-wall (sparing subendocardial portion) enhancement with non-ischemic distribution and with a patchy distribution, normally localized in the basal and mid-ventricular segments of the posterolateral wall[13]. Lake Louise Consensus criteria include three CMR features for improving diagnostic accuracy: 1) increased regional or global signal on $\mathrm{T} 2$-weighted images; 2) increased early gadolinium enhancement ratio (between myocardium and 
skeletal muscle), 3) one or more focal lesions of LGE without an ischemic distribution. The fulfilment of two or more criteria have a diagnostic accuracy can of 78-85\% compared with endomyocardial biopsy[7]. Moreover, the segmental distribution of LGE findings can aid for a successful subsequent endomyocardial biopsy, and there is a good diagnostic agreement between CMR and endomyocardial biopsy for the diagnosis of myocarditis. Edema and early
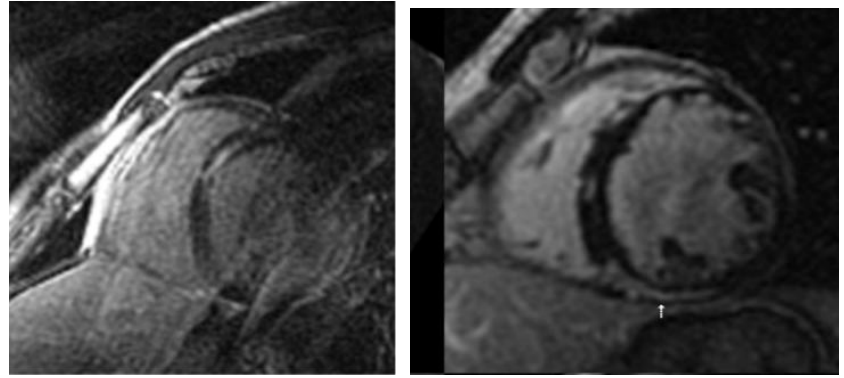

Figure4. LGE sequence showing a non-ischemic subepicardial pattern in the antero-inferior wall of the left ventricle (down), and a midwall pattern in the anterosuperior wall), corresponding to a myocarditis.

\section{Pericarditis (Figure 5)}

CMR is the best imaging method for the diagnosis and management of pericarditis. Acute pericarditis manifests with pericardial thickening and pericardial effusion. LGE sequence shows intense focal or diffuse pericardial enhancement that correlates well with the inflammatory state. This fact makes LGE very useful to guide the tapering of the anti-inflammatory therapy. In pericardial
LGE resolve with resolution of acute myocarditis whereas persistent LGE (> 4 weeks) indicates irreversible fibrotic injury, because viral clearance occurs in few days after infection and myocardial inflammation usually clears by 2-3 weeks. LGE also has been found to be the best independent predictor of overall and cardiovascular myocarditis $[9,13]$.

constriction, a complication secondary to chronic pericarditis that requires pericardiectomy as the definitive treatment, if CMR shows LGE as evidence of pericardial inflammation the constriction might be reversible with aggressive anti-inflammatory therapy, thus obviating the need for surgery $(14,15)$. So LGE have important diagnostic and therapeutic implications in the context of pericarditis.

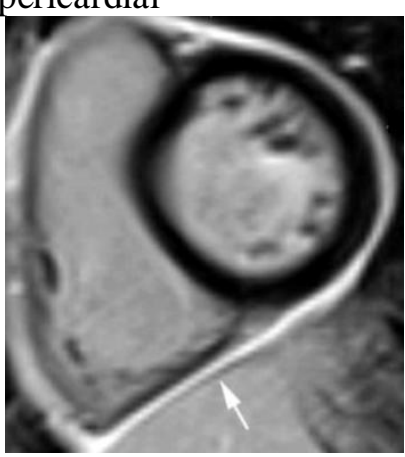

Figure5. LGE sequence showing global pericardial enhancement that indicates active pericardial inflammation in the context of an acute pericarditis.

\section{KAWASAKI DiSEASE}

Cardiovascular manifestations, especially coronary artery aneurysms, are common and are the leading cause of long-term morbidity and mortality. Myocarditis is practically universal in the acute phase of the disease and responds well to intravenous immunoglobulin without development of fibrosis. Myocardial infarction can develop in the setting of large coronary aneurysms. CMR with LGE sequence is a valuable non-invasive method for simultaneous evaluation of coronary arteries anatomy, cardiac function, myocardial inflammation in the acute phase and fibrosis in the chronic follow-up $[2,11,16,17]$.

\section{CARDIOMYOPATHIES}

\subsection{Dilated Cardiomyopathy (DCM)}

It is the most common type of cardiomyopathy in children. It is characterized by ventricular chamber enlargement and systolic dysfunction with normal or reduced left ventricular wall thickness, with no identifiable coronary artery disease. Most of cases (69\%) are idiopathic, but 
other etiologies include myocarditis and inborn errors of metabolism. Histologically the findings are non-specific and include myocytes hypertrophy and fibrosis usually in the base and mid-ventricular regions. When LGE is seen (30\% in adults), the typical pattern is in form of linear stripes and patches in the basal to midventricular regions in a non-vascular distribution (non-ischemic pattern)(14,20). In children, LGE has been showed in only $16 \%$ possibly due to a different repair mechanism for fibrosis in children that enables favourable reverse remodelling[4,7,9]. LGE has important prognostic implications because the presence of fibrosis is related with an increased likelihood of all cause mortality and also of malignant arrhythmias; therefore LGE may be a hopeful advancement in the risk stratification and management of these children (ICD implantation).

\subsection{Hypertrophic Cardiomyopathy (HCM; Figure 6)}

$\mathrm{HCM}$ is the most common genetic cardiomyopathy (incidence 1/500) and the most common cause of sudden death in the young. CMR provides a complete description of abnormalities in HCM, including morphology, mass, function, haemodynamic obstruction and tissue characterization. LGE provides evidence for myocardial fibrosis that corresponds with myocardial disarray detected in necropsies in patients with HCM. LGE is present in 28-73\% of children with HCM in a similar pattern as in adults. [6,9]. The typical pattern of LGE usually involves the septum and includes focal areas of bright signal in hypertrophied regions or small patches close to the superior and inferior right ventricular insertion points. The presence of LGE in patients with HCM has an important prognostic significance, since it has been reported as an increased risk for sudden cardiac death, systolic dysfunction and non-sustained ventricular tachycardia compared with patients without LGE in CMR. Therefore LGE sequence may have an important role in improving the risk assessment in borderline or controversial cases benefiting from ICD[6,7].

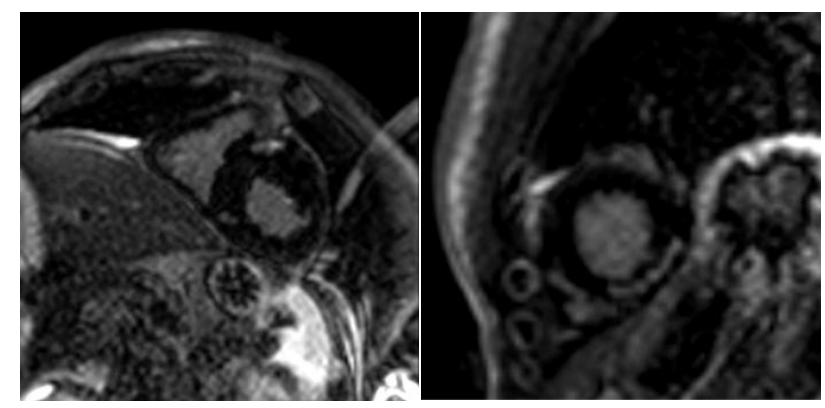

Figure6. LGE sequence showing HCM with midmyocardial hyperenhancement at the insertion points of right ventricle (up), and a mid wall pattern in the postero inferior wall of left ventricle (Fabry disease).

Anderson-Fabry Disease: It is storage disease due to the deficiency of $\alpha$-galactosidase that produces intracellular accumulation of glycosphingolipid in different tissues. Cardiac involvement presents with concentric HCM, heart failure and arrhythmias. Distinguishing Fabry disease from HCM (up to $6 \%$ of suspected HCM are Anderson-Fabry disease) is important because the former is managed by enzyme replacement therapy with improvement in LV function, and the latter by pharmacological therapy/surgery/ICD $[4,7,9]$. LGE imaging is present in $50 \%$ of the cases and can help differentiate Fabry disease from HCM. In Fabry disease LGE presents typically a midmyocardial or subepicardial pattern in the basal inferolateral wall. This is a key point since the isolated involvement of inferolateral wall is rare in $\operatorname{HCM}[18,19]$. CMR is also useful in monitoring response to enzyme replacement therapy, which shows decreased left ventricular mass, and can provide the adequate site for biposy.

Left Ventricular Non-Compaction: Left ventricular non-compaction is a rare condition secondary to an arrested embryogenesis that results in the presence of a 'spongy' appearance of the LV myocardium, commonly involving the anterior and lateral walls, and characterised by increased trabeculation and intratrabecular recesses in the myocardium. The diagnosis is made by the presence of exaggerated ventricular trabeculations, with a ratio of non-compacted to compacted myocardium $>2.3$ in diastole at echocardiography or CMR[14,20,21]. LGE is present in $25-40 \%$ of the patients including different patterns (subendocardial (70\%), 
midwall (11\%) and transmural (15\%) enhancement)[4,21], and its presence is associated with a worse left ventricular ejection fraction and the development of ventricular arrhythmias.

Muscular Dystrophies: Muscular dystrophies (Duchenne and Becker disease) are X-linked neuromuscular disorders caused by abnormal dystrophin, which results in necrosis of cardiac and skeletal muscle fibers and their replacement with connective tissues and fat. Cardiac abnormalities are the most common cause of death in these children, and include DCM, heart failure, arrhythmia and sudden death. LGE has been reported in 32-89\% of Duchenne cases, following usually a subepicardial or midmyocardial pattern at the free wall of the left ventricle. $[4,13]$. LGE is progressive and may become transmural in severe cases and can indicate cardiac involvement before the presence of cardiac symptoms or electrocardiographic or echocardiographic abnormalities. LGE have important prognosis implications because it is related to lower left ventricular ejection fraction and death. LGE can also guide the timing for pacemaker/ICD placement $[4,13]$.

\section{Arrhythmogenic Right Ventricular} Dysplasia/Cardiomyopathy (ARVD/C): is a rare inherited cardiomyopathy characterized by replacement of right ventricle myocardium with fibro-fatty tissue leading to arrhythmias, progressive right ventricular dysfunction and sudden cardiac death. LGE is pre sent in only $13 \%$ of children with ARVD/C, usually following a midwall pattern at the inferolateral wall of right ventricle in the classic form, but left ventricle also can be involved. The low incidence of LGE in children suggests a limited value for diagnosis in early stages of disease in children[9,13]. Although LGE is not part of the proposed diagnostic criteria, when present it had excellent correlation with fibro-fatty changes on endomyocardial biopsy and is associated with ventricular arrhythmias, so LGE has a prognostic value in this setting.

\section{Congenital Heart Diseases}

Congenital heart diseases are the most frequent cardiac disorders in children. In these pathologies the following factors can coexist to produce myocardial fibrosis: 1) Acute or chronic ischemic damage caused by prolonged states of hypoxemia in the case of uncorrected cyanotic congenital heart disease (Tetralogy of Fallot, Transposition of great arteries...), alterations in the anatomy or coronary flow (congenital coronary anomalies isolated or associated with other congenital heart diseases), corrective surgery that involves the manipulation of the coronary arteries (Jatene technique for Transposition of great arteries), or volume/pressure overload (aortic stenosis, hearts univentricular hearts in Fontan phase, pulmonary insufficiency in corrected tetralogy of Fallot ...) 2) Scars caused by surgical incisions, sutures, or implantation of prosthetic material (patches ...). LGE is usually located at the site of surgical manipulation (Figure 7) but occasionally extend beyond the surgical site, and increases with the age. The most important thing about the LGE in congenital heart diseases is that the LGE extension has prognosis implications because it is usually associated to ventricular dilatation, ventricular low ejection fraction, low exercise capacity, ventricular arrhythmias and sudden cardiac death. Because of the above the presence of myocardial fibrosis is expected throughout the course of a patient with congenital heart disease, therefore the LGE sequence is very useful in the follow-up of these patients $[22,23]$.

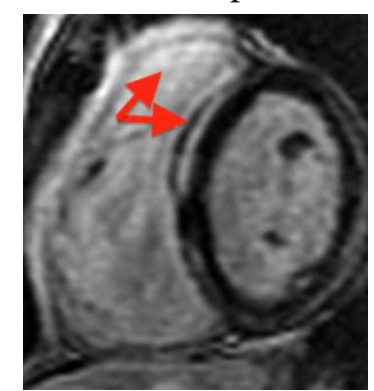

Figure7. LGE sequence showing enhancement located at right ventricular outflow tract in a patient with a corrected Tetralogy of Fallot.

\section{Cardiac Masses (Figure 8)}

CMR is one of the better image techniques for the evaluation of cardiac masses. Due to its tissue characterization capabilities, it can make the differential diagnoses of cardiac masses without the need of avoiding confirmatory biopsy in some cases. It is also valuable in differentiating thrombus from neoplasm. A 
thrombus has low signal intensity in LGE images and does not show enhancement unless it is vascularized in chronic stages[8,23]. Rhabdomyoma, the most common benign pediatric cardiac neoplasm (60\%) usually does not present LGE, while fibroma, the second most common primary tumor in children, metastasis and sarcoma (the most common primary cardiac malignancy in children (95\%) usually shows heterogeneous LGE [4,9].
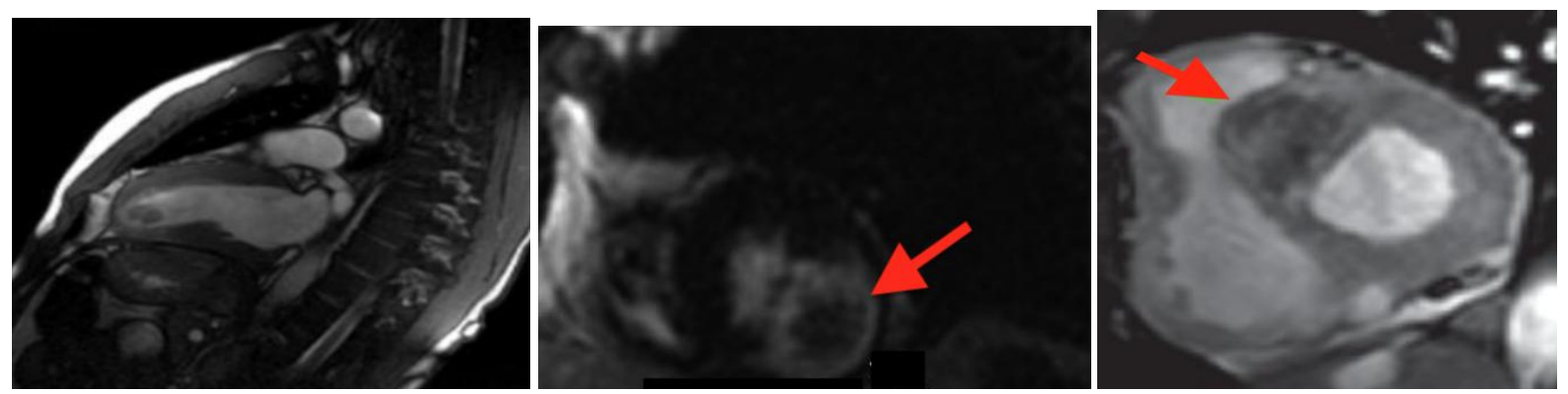

Figure8. LGE sequences showing a LV apical thrombus with no enhancement (up), a cardiac rabdomioma with no enhancement (middle), and a cardiac fibroma with heterogeneous enhancement.

\section{CONCLUSION}

The ability to characterize cardiac tissues in a non-invasive way has converted CMR with LGE sequence into an essential diagnostic tool for the assessment of a wide and heterogeneous range of heart diseases in children. The presence, pattern and extension of LGE may aid in the diagnosis of underlying pathology and remarkably offers information regarding viability and prognosis. However, pediatric CMR remains technically challenging with limitations due to small size, high heart rates, and motion artefacts from cardiac pulsation, respiration, and technical or procedural difficulties inherent to infants. Also mixed cardiological and radiological expertise is needed. The challenges lie in developing new technological advances that facilitate the LGE assessment approach into clinical routine practice.

\section{REFERENCES}

[1] Bonnemains, L., Raimondi, F. \& Odille, F. Specifics of cardiac magnetic resonance imaging in children. Archives of Cardiovascular Diseases 109, 143-149 (2016).

[2] Goo, H. W. Coronary Artery Imaging in Children. Korean J Radiol 16, 239-12 (2015).

[3] Latus, $H$. et al. Cardiovascular magnetic resonance assessment of ventricular function and myocardial scarring before and early after repair of anomalous left coronary artery from the pulmonary artery. Journal of Cardiovascular Magnetic Resonance 16, 1-9 (2014).

[4] Etesami, M., Gilkeson, R. C. \& Rajiah, P. Utility of late gadolinium enhancement in pediatric cardiac MRI. Pediatr Radiol. 46, 1096-1113 (2016).
[5] Green, J. J., Berger, J. S., Kramer, C. M. \& Salerno, M. Prognostic Value of Late Gadolinium Enhancement in Clinical Outcomes for Hypertrophic Cardiomyopathy. JAAC: Cardiovascular Imaging 5, 370-377 (2012).

[6] Asif. Emerging Concepts for Myocardial Late Gadolinium Enhancement MRI. Current Cardiology Reviews. 9, 185-190, (2013).

[7] Sorensen, C. et al. Cardiac CT or MRI in pediatric practice: Which one to choose? Diagnostic and Interventional Imaging 97, 513517 (2016).

[8] Vermes, E., Carbone, I., Friedrich, M. G. \& Merchant, N. Patterns of myocardial late enhancement: Typical and atypical features. Archives of Cardiovascular Diseases 105, 300 308 (2012).

[9] De Roos, A. \& Higgins, C. B. Cardiac Radiology: Centenary Review. Radiology. 273, S142-S159 (2014).

[10] Newburger, J. W., Takahashi, M. \& Burns, J. C. Kawasaki Disease. Journal of the American College of Cardiology 67, 1738-1749 (2016).

[11] Printz, B. F. Noninvasive imaging modalities and sudden cardiac arrest in the young: can they help distinguish subjects with a potentially life-threatening abnormality from normals? Pediatric Cardiology 33, 439-451 (2012).

[12] Mahrholdt, H., Wagner, A., Judd, R. M., Sechtem, U. \& Kim, R. J. Delayed enhancement cardiovascular magnetic resonance assessment of non-ischaemic cardiomyopathies. European Heart Journal 26, 1461-1474 (2005).

[13] Saeed, M. Magnetic resonance imaging and multi-detector computed tomography assessment of extracellular compartment in ischemic and non-ischemic myocardial pathologies. World Journal of Cardiology. 6, 1192-18 (2014). 
[14] Chadi Alraies, M., et al. Usefulness of Cardiac Magnetic Resonance-Guided Management in Patients with Recurrent Pericarditis. American Journal of Cardiology. 115, 542-547 (2015).

[15] Cantarini, L. et al. Autoimmunity and autoinflammation as the yin and yang of idiopathic recurrent acute pericarditis. Autoimmunity Reviews 14, 90-97 (2015).

[16] Johnson, P. T. \& Fishman, E. K. CT Angiography of Coronary Artery Aneurysms: Detection, Definition, Causes, and Treatment. American Journal of Roentgenology 195, 928934 (2010).

[17] Dehaene, A., Jacquier, A., Falque, C., Gorincour, G. \& Gaubert, J. Y. Imaging of acquired coronary diseases: From children to adults. Diagnostic and Interventional Imaging 97, 571-580 (2016).

[18] Moosa, S. \& Ntusi, N. A. B. Role of cardiovascular magnetic resonance in the evaluation of cardiomyopathy. South Africa Journal of Radiology. 20, 402-10 (2016).
[19] Serbin, V. I. \& Khaĭdarov, B. M. Hypertrophic cardiomyopathy in children. Pediatriia 17, 6871 (1986).

[20] Udeoji, D. U., Philip, K. J., Morrissey, R. P., Phan, A. \& Schwarz, E. R. Left ventricular noncompaction cardiomyopathy: updated review. Ther Adv Cardiovasc Dis 7, 260-273 (2013).

[21] Taylor, A. M. Cardiac imaging: MR or CT? Which to use when. Pediatr Radiol 38, 433-438 (2008).

[22] Friedberg, M. K. et al. Impaired right and left ventricular diastolic myocardial mechanics and filling in asymptomatic children and adolescents after repair of tetralogy of Fallot. European Heart Journal: Cardiovascular Imaging. 13, 905-913 (2012).

[23] Dacher, J. N. et al. CT and MR imaging in congenital cardiac malformations: Where do we come from and where are we going? Diagnostic and Interventional Imaging. 97, 505-512 (2016).

Citation: Moises Rodriguez-Gonzalez, Ana Castellano-Martinez. Clinical Applications of Late Gadolinium in Children. ARC Journal of Pediatrics. 2018; 4(1): 1-8. doi:dx.doi.org/10.20431/2455-5711.0401001.

Copyright: (C) 2018 Authors. This is an open-access article distributed under the terms of the Creative Commons Attribution License, which permits unrestricted use, distribution, and reproduction in any medium, provided the original author and source are credited. 